\title{
I have an arrhythmia. Do I need Cardiac Rehabilitation ?
}

\section{ZGIIA Alexandra ${ }^{1}$, MAATAOUI Fadi ${ }^{1}$, CISMARU Gabriel ${ }^{1}$, CISMARU Andrei ${ }^{2}$, GURZAU Diana, FRANGU Florina ${ }^{1}$, MARTIS Alexandru ${ }^{1}$, CALOIAN Bogdan ${ }^{1}$, COMSA Horatiu ${ }^{1}$, GUSETU Gabriel $^{1}$, PUIU Mihai ${ }^{1}$, Istratoaie Sabina ${ }^{3}$, ROSU Radu ${ }^{1}$, POP Dana ${ }^{1}$, ZDRENGHEA Dumitru ${ }^{1}$}

Corresponding author: Gabriel CISMARU, E-mail: gabi cismaru@yahoo.com

1. 5th Department of Internal Medicine, Cardiac Rehabilitation, "Iuliu Hatieganu" University of Medicine and Pharmacy, Cluj-Napoca, Romania.

2. Research Center for functional Genomics, Biomedicine and Translational Medicine, "Iuliu Hatieganu" University of Medicine and Pharmacy, Cluj-Napoca, Romania.

3. Department of Pharmacology, Toxicology and Clinical Pharmacology, "Iuliu Haţieganu" University of Medicine and Pharmacy, Cluj-Napoca, Romania.

\begin{abstract}
This review shows that cardiac rehabilitation is not contraindicated in patients with arrhythmias; furthermore, it may be beneficial in reducing the number and severity of arrhythmia episodes. We will briefly describe the most common cardiac arrhythmias for which there is information in the medical literature related to inclusion in a rehabilitation program: inappropriate sinus tachycardia, atrial fibrillation, premature atrial contractions, atrial flutter, premature ventricular beats, ventricular tachycardia. All these data support the use of cardiac rehabilitation for patients with arrhythmia even though this is underused and underappreciated by many clinicians.
\end{abstract}

Key words: cardiac rehabilitation, arrhythmia, atrial fibrillation, ventricular premature beats, ventricular tachycvardia, atrial premature beats, physical activity,

\section{Introduction}

Cardiac rehabilitation has been shown to be effective in patients with a history of myocardial infarction, percutaneous coronary intervention and aortocoronary bypass $(1,2,3)$. For these reasons, current European and American guidelines recommend cardiac rehabilitation for all these categories of patients (4). On the other hand, being included in a cardiac rehabilitation program not only improves the individual's physical capacity and physical quality, but has also been shown to decrease mortality by decreasing the number and severity of arrhythmic events (5).

Cardiac arrhythmias, depending on their type, from the simplest to the most severe, can have consequences on the patient's life: from panic attack to heart failure, stroke and even arrhythmic death. In addition to antiarrhythmic treatment and catheter ablation, the cardiologist should consider lifestyle changes. Because physical inactivity is an essential risk factor for cardiovascular disease, it should be borne in mind that, with the recommendations of antiarrhythmics, cardiac rehabilitation should also be recommended, with a progressive increase in physical activity. Both adults and old patients (6) benefit from rehabilitation $(7,8,9)$.

Rehabilitation can also be performed in patients with implanted devices: pacemakers, ICDs, CRTs $(10,11)$. In Romania there are hospitals and centers that can perform cardiac rehabilitation, and arrhythmias should be one of the indications for starting rehabilitation.

This review will show that cardiac rehabilitation is not contraindicated in patients with arrhythmias; furthermore, it may be beneficial in reducing the number and severity of arrhythmia episodes. We will briefly describe the most common cardiac arrhythmias for which there is information in the medical literature related to inclusion in a rehabilitation program.

Certainly, cardiac rehabilitation will be delayed in patients with severe sustained ventricular tachycardia or ventricular fibrillation, unstable angina or acute myocardial infarction, severe hypertension, intracardiac ventricular thrombosis or pedicles until the status of the patient permits physical activity. 


\section{1. Inappropriate sinus tachycardia.}

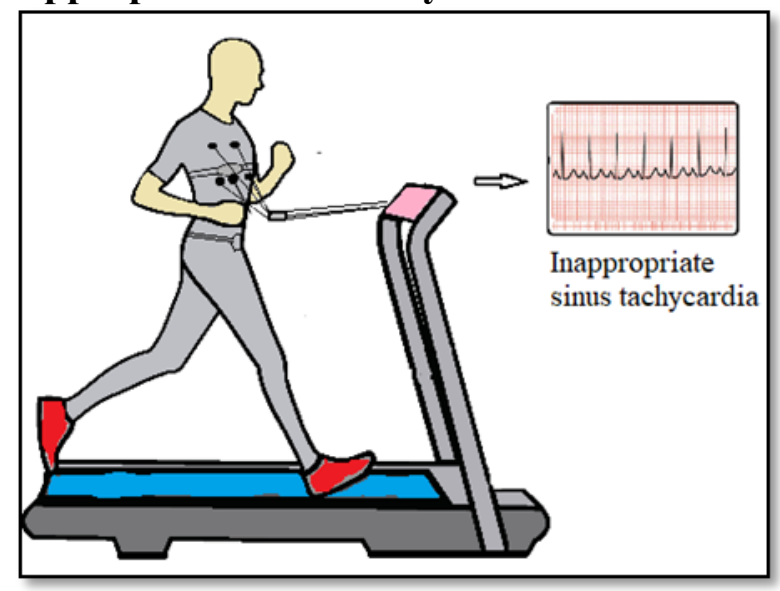

It is a form of arrhythmia characterized by an excessive increase in the sinus rate in response to low intensity effort. It is defined as a resting heart rate of over $100 \mathrm{bpm}$ or an average heart rate $>90$ bpm during 24-hour Holter ECG in the absence of reversible causes such as hyperthyroidism, anemia, fever, hypovolemia, hypoxia, central nervous system stimulation, diabetic dysautonomia, etc. In inappropriate sinus tachycardia the $\mathrm{P}$ wave has a sinus morphology in the 12-lead electrocardiogram. One of the mechanisms that explain arrhythmia is the increase of sinus node automaticity. The treatment methods of inappropriate sinus tachycardia include: antiarrhythmic drugs, catheter ablation and cardiac rehabilitation.

The rehabilitation of patients with inappropriate sinus tachycardia implies the following general measures: 1) sleeping with the head raised to 30 degrees, 2) proper hydration, 2-2.5 liters/day, 3) endurance training, 5) back pressure maneuvers and 6) proper salt consumption, above $10-20 \mathrm{mg}$ /day. 7) Elastic compression stockings are useful in patients with venous insufficiency of the lower limbs (12).

Before starting the cardiac rehabilitation program, tests are carried out to evaluate the sympathetic/parasympathetic autonomic system: 1) the cold water test (also used in patients with paroxysmal supraventricular tachycardia as a method of treatment) induces bradycardia by activating the parasympathetic system during application of cold water on the face, which sends efferent signals to the cardiac parasympathetic ganglia. In patients with inappropriate sinus tachycardia, the response is abnormal with the appearance of tachycardia instead of bradycardia; 2) the isoprenaline test - which shows an excessive increase in heart rate after its administration; 3) evaluation of the intrinsic heart rate after administration of propranolol and atropine (which blocks sympathetic and parasympathetic efferent signals to the heart), which confirms the increase of sinus node automaticity; and 4) the response to the Valsalva maneuver, which shows an abnormal relationship between the maximum heart rate and the minimum heart rate recorded during the maneuver. After completing the cardiac rehabilitation program, all these values are checked again and compared with those recorded at the beginning of the training program (13).

The Levine program was proposed for patients with inappropriate sinus tachycardia and orthostatic postural tachycardia. It includes 3 months of physical training associated with lifestyle changes: sleeping at 30 degrees head tilt, water intake 3 liters/day and salt intake 10 grams/day. Both before and after cardiac rehabilitation, the patients are tested for sympathetic/parasympathetic imbalance (13).

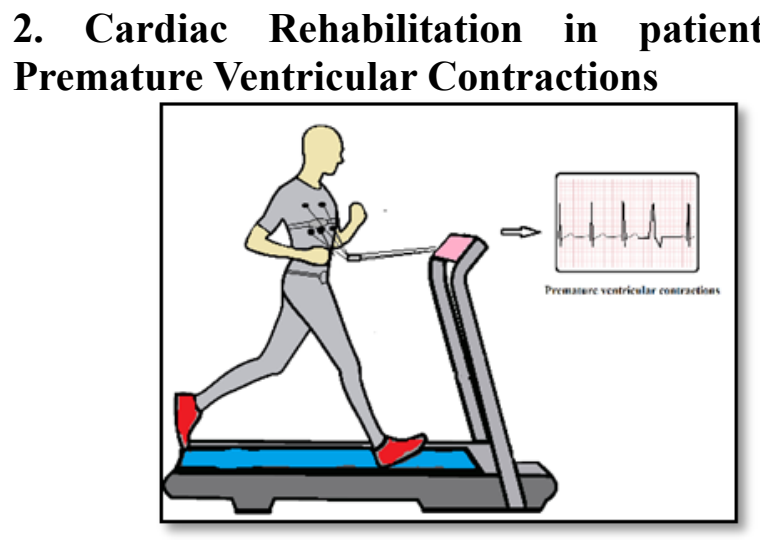

Premature ventricular contractions are present in more than $6 \%$ of middle-age adults. Their incidence increase with age, low potasium or magnesium levels, history of structural heart disease, and hypertension. There are studies on patients with PVCs that performed cardiac rehabilitation. Lillis et al verified the effect of physical training during cardiac rehabilitation on the the number of ventricular premature contractions in 18 patients performing included in a cardiac rehabilitation program. Patients with stable ischemic heart disease were divided into two groups: 9 with a high risk of cardiovascular events and 9 with low risk. All 18 patients were followed by Holter EKG during physical exertion as well as during resting period. Physical exercise was performed on ergometric bicycle or treadmill, 40 minutes daily at an intensity of $75 \%$ of maximum METS or $85 \%$ of the maximum theoretical heart rate. 
Results showed that during the days with physical training, the number of ventricular premature contractions in Holter EKG was higher than during resting days. Compared to the low risk group, in the high risk group the number of premature ventricular contractions was higher during effort or immediately after stopping the effort. During sleep or during sexual activity, the number of ventricular premature contractions did not increase. Patients in both groups were followed for 7.5 years and no deaths were recorded in any of the groups (14).

The study of Boukhris et al. on 122 patients with 59 diabetic and 63 non-diabetic patients, demonstrated 2 essential things: 1) cardiac rehabilitation in diabetic patients improves repolarization parameters (corrected QT interval and QT interval dispersion) and 2) the number of ventricular premature contractions evaluated by Holter EKG in both diabetic and non-diabetic patients decreased at the end of the rehabilitation program. In addition, the severity of ventricular premature contractions expressed by the Lown class decreased at the end of the rehabilitation program in both diabetic and nondiabetic cat patients (15).

\section{Cardiac rehabilitation in patients with Ventricular Tachycardia and Ventricular Fibrillation}

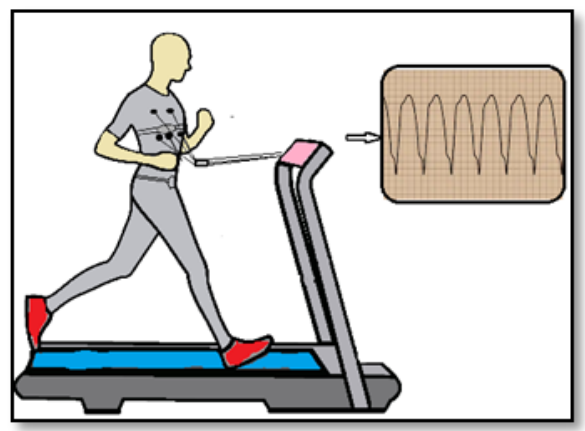

Patients with ventricular tachycardia and fibrillation may undergo cardiac rehabilitation, obviously outside the acute episode, when the patient is free of ventricular arrhythmias.

Even after repeated episodes of ventricular tachycardia or ventricular fibrillation, cardiac rehabilitation can be performed. This is the case of patients with electrical storm. Electrical storm is defined as frequent episodes of ventricular tachycardia or ventricular fibrillation occurring within a short period of time. Obviously it is a lifethreatening arrhythmia and the treatment consists of antiarrhythmic drugs or catheter ablation.
It is very important after an episode of electrical storm to prevent the occurrence of recurrences and an important place is occupied by cardiac rehabilitation. It increases the physical capacity, allows daily activity, and for hospitalized patients, allows safely discharge (4).

The study of Kato et al. evaluated the feasibility of cardiac rehabilitation in patients with electrical storm. The study included 67 patients divided into 2 groups: the cardiac rehabilitation group (which included 39 patients) and the non-rehabilitation group (which included 28 patients). The results showed: regarding the recurrences of electrical storm between the 2 groups, the percentages were not statistically different $(13 \%$ vs. $21 \%)$. Furthermore, the number VT and VF episodes were similar between the 2 groups $(28 \%$ vs. $25 \%)$. However, mobilization within the first 48 hours after the electric storm had adverse cardiac effects: recurrent electric storm $(21 \%$ vs. $6 \%)$ and recurrent VT / VF (34\% vs. 19\%) in the early mobilization group compared to the late mobilization group. Furthermore, early mobilization within the first 48 hours was associated with higher BNP values, and lower ejection fraction. On the other hand, in the group without cardiac rehabilitation there were 3 deaths by uncontrolled ventricular arrhythmias, in contrast to no deaths in the group undergoing cardiac rehabilitation. Another important result was that daily physical activity significantly improved in the cardiac rehabilitation group (16)

\section{4. Cardiac rehabilitation in patiens with atrial premature contractions.}

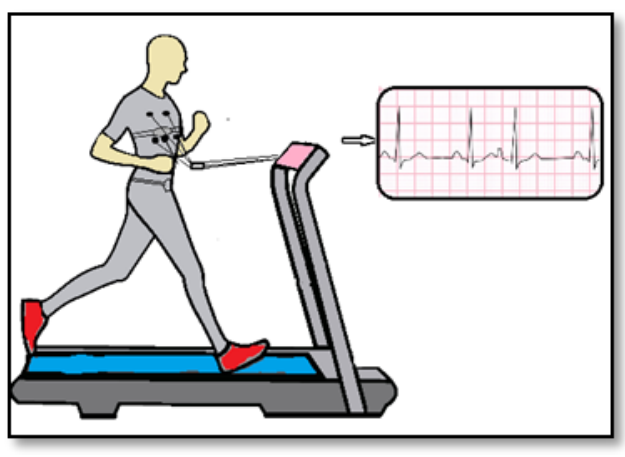

Premature atrial contractions are common arrhythmias and are found both in patients who want to start a cardiac rehabilitation program and in patients who perform exercise training. Physical exercise leads to an increased plasma concentration of circulating catecholamines, which may be an inducing factor for the occurrence of premature 
atrial contractions. For these reasons, the first line of treatment in this case is the administration of beta blockers. It is also well known that cardiac rehabilitation decreases the plasma concentration of adrenaline and noradrenaline. For these reasons, rehabilitation can be performed and is totally indicated in patients with premature atrial contractions.

Younis et al. included 213 patients with premature atrial contractions in a 6-month cardiac rehabilitation program, consisting of 2 sessions/week of exercise, lasting 60 minutes. Premature atrial contractions were present before inclusion in the rehabilitation program, being revealed during an initial stress test. At long-term follow-up it was confirmed that patients with premature atrial contractions had a 2-fold increased risk of atrial fibrillation. On the other hand, the incidence of major cardiac adverse effects and mortality was similar in the group with premature atrial contractions and in the group without premature atrial contractions (17).

\section{Cardiac rehabilitation in patients with Atrial fibrillation}

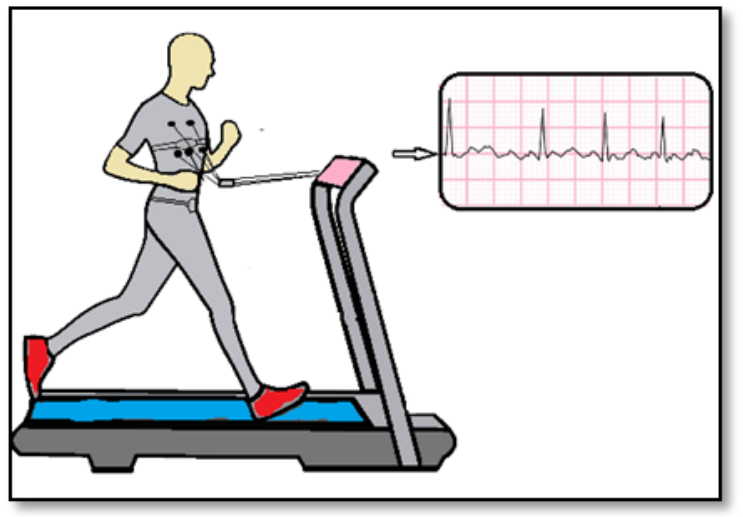

Atrial fibrillation is the most frequent cardiac arrhythmia. Therefore, patients who already had an episode of AF should be advised regarding their physical, psychological and social life. Thus, the cardiologist makes recommendations on the physical exercise and lifestyle modifications in order to stabilize, slow down or even reverse the underlying arrhythmic process. Patients with both paroxysmal and persistent/permanent atrial fibrillation can be re-included in socio-professional and family life (18).
Since the 1990s, the problem of an increased risk of atrial fibrillation has been raised in individuals practicing high intensity endurance sports. Endurance sports can precipitate dilation of the left atrium, hypertrophy of the left ventricle and alterations of the autonomic cardiac nervous system. On the other hand, moderate intensity sport has been shown to lower blood pressure levels, decrease left ventricular hypertrophy, and reduce dilation of the left atrium. Furthermore, the Legacy study showed that moderate intensity sports lead to weight loss, and indirectly reduce the number of episodes of atrial fibrillation and also the length of AF episodes. The results are similar to those of Mozaffarian et al., which confirm that moderate physical activity in patients aged over 65 years reduces the risk of atrial fibrillation by $28 \%$ compared to patients who do not practice sports. At the same time, a study published by Azarbal et al. on an impressive number of $>$ 90,000 women with a mean age of 63 years showed that physical exertion of 15 MET x hour leads to a significant decrease in AF episodes compared to women who practiced $<1$ MET x hour (19).

Depending on the type of atrial fibrillation, the way cardiac rehabilitation is performed differs from case to case. In patients with the paroxysmal (or nonpermanent) form, the role of rehabilitation is to prevent other episodes of AF, whereas in patients with the permanent form, the objective is to control heart rate. The same goal is that of drug treatment in patients with paroxysmal and permanent AF, so cardiac rehabilitation is actually a substitute for medication.

In the study of Mozaffarian, 51 patients with nonpermanent $\mathrm{AF}$ were included and divided into 2 groups: the cardiac rehabilitation group and the control group. Interestingly, patients in both groups were monitored by a loop recorder, all of them agreeing with the subcutaneous implantation of the monitoring device. The monitored period was 4 weeks before rehabilitation and 4 weeks after completion of the cardiac rehabilitation program. As a result, in the rehabilitation group the duration of AF episodes decreased from $8.1 \%$ to $4.6 \%$, while in the control group it increased from $10.4 \%$ to $14.6 \%$, the difference being statistically significant. In addition to these results, an important benefit was observed on the left ventricular systolic function evaluated by echocardiography, quality of life and lipid profile after completion of the rehabilitation program (19). 
In the CARDIO-FIT study, conducted in Adelaide, Australia, patients with non-permanent atrial fibrillation were included, all of them obese with a BMI $>27 \mathrm{~kg} / \mathrm{sqm}$. Initially, low intensity physical activity was recommended for 20 minutes, 3 times per week, which was gradually increased to 200 minutes of moderate intensity/week. The maximum heart rate allowed during exercise was $85 \%$ of the theoretical maximum heart rate (220-age), and the patients were advised to avoid reaching this rate. A treadmill stress test was performed, using Bruce's protocol, before the inclusion in the program and at the end of the rehabilitation program. In patients whose cardiac rehabilitation had a beneficial effect defined as $>2$ MET improvement, there was a significant decrease in weight, a significant decrease in blood pressure and the number of hypotensive drugs used, a significant decrease in cholesterol, triglycerides and hypolipidemic drugs, glycosylated hemoglobin, blood glucose, a decrease in highsensitive C-reactive protein (hs-CRP), a decrease in the number and duration of $\mathrm{AF}$ episodes and a decrease in the number of AF recurrences after conversion to sinus rhythm or catheter ablation. The authors conclude that each MET of physical capacity improvement leads to a $20 \%$ decrease in $\mathrm{AF}$ recurrences in obese patients (20).

In patients with permanent atrial fibrillation, circumstances are different because it is no longer possible to prevent recurrences or decrease the duration of AF episodes. The goal is to decrease the heart rate during rest and effort and to reduce the need of antiarrhythmic drugs. Osbak et al. published in 2011 a study on 49 patients with permanent AF which were included in a cardiac rehabilitation program. The program consisted of 12 weeks of internment training composed of 3 sessions $x 1$ hour per week training. Each session contained: heating, high intensity training and deceleration or gradual return to rest. The results showed a higher intensity reached during the stress test in the rehabilitation group compared to the control group, and a longer distance for the 6 minute walk test, with a decrease of the resting heart rate. At the same time, the quality of life improved in the cardiac rehabilitation group, demonstrating the beneficial effects of cardiac rehabilitation in patients with permanent atrial fibrillation (21).
Another randomized study of 30 patients with permanent AF showed an improvement of the exercise capacity and heart rate variability after 2 months of training. Pliene et al. studied the change of heart rate in 10 patients with permanent $\mathrm{AF}$ who underwent cardiac rehabilitation with moderate physical training (45 minutes of walking or jogging 2 times a day). They demonstrated that physical training controls heart rate in patients with permanent AF similarly to beta blockers and digoxin, inducing a decrease in the mean heart rate on Holter ECG from $76 / \mathrm{min}$ to $67 / \mathrm{min}$ (approximately 12\%) (22).

\section{Cardiac rehabilitation in patients with Atrial Flutter}

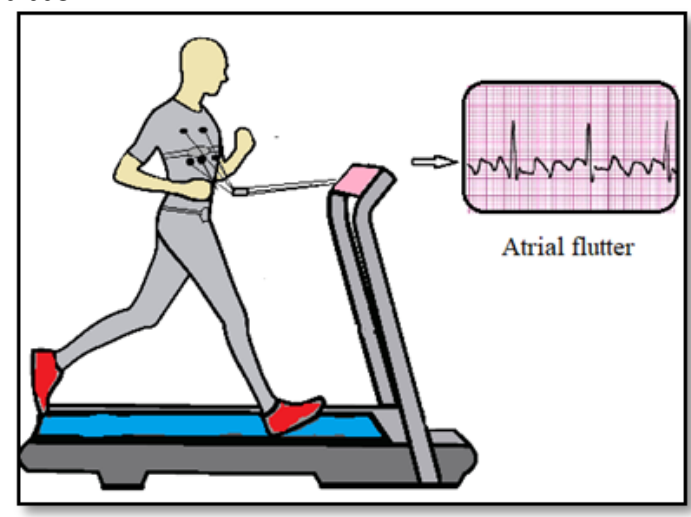

There are few studies examining the effect of exercise training in patients with atrial flutter. Most of the studies are performed in patients with atrial fibrillation but also include patients with atrial flutter in the analysis, because of the similarity between the 2 arrhythmias.

A Norwegian study followed up endurance athletes over a 10-year period and described the number of individuals who developed atrial flutter during this period (23). Out of 3545 men, 52 had atrial flutter. On the other hand, there was a dose-response relationship between the total duration of endurance sports and the risk of developing atrial flutter in men over 53 years. Thus, the authors declare that this is the first study to establish a dose-response link between endurance training and atrial flutter. One of the strengths of this study was that investigators were able to differentiate between atrial fibrillation and atrial flutter based on ECG recording. Even if the patients had similar symptoms, differentiation is still possible due to the morphology of the atrial $\mathrm{P}$ wave on the 12 lead ECG. 


\section{Conclusion:}

Cardiac rehabilitation is a valuable treatment for a large spectrum of patients with cardiac arrhythmias. Its benefits are based on research that demonstrates a decrease in the number and duration of arrhythmia episodes, an improvement in quality of life, psychological status, and a decrease in morbidity and mortality. It also reduces the hospitalization duration and readmission. All these data support the use of cardiac rehabilitation for patients with arrhythmia even though this is underused and underappreciated by many clinicians.

\section{References:}

1. Malfatto G, Facchini M, Bragato R, Branzi G, Sala L, Leonetti G. Short and long term effects of exercise training on the tonic autonomic modulation of heart rate variability after myocardial infarction. Eur Heart J. 1996; 17:532-538.

2. Oldridge NB, Guyatt GG, Fischer ME, Rimm AA. Cardiac rehabilitation after myocardial infarction: combined experience of randomized clinical trials. J Am Med Assoc. 1988;260:945-950.

3. O'Connor GT, Buring JE, Yusuf S, et al. An overview of randomized trial of rehabilitation with exercise after myocardial infarction. Circulation. 1989;80: 234-244.

4. Perk J, De Backer G, Gohlke H, et al. European Guidelines on Cardiovascular Disease Prevention in Clinical Practice (version 2012). The Fifth Joint Task Force of the European Society of Cardiology and other societies on cardiovascular disease prevention in clinical practice. Eur $\mathrm{J}$ Prev Cardiol. 2012;19(4):585e667. 5. Thomas RJ, King M, Lui K, et al, AACVPR, ACC, AHA, American College of Chest Physicians, American College of Sports Medicine, American Physical Therapy Association, Canadian Association of Cardiac Rehabilitation, European Association for Cardiovascular Prevention and Rehabilitation, Inter-American Heart Foundation, National Association of Clinical Nurse Specialists, Preventive Cardiovascular Nurses Association, Society of Thoracic Surgeons. AACVPR/ACC/ AHA 2007 performance measures on cardiac rehabilitation for referral to and delivery of cardiac rehabilitation/secondary prevention services endorsed by the American College of
Chest Physicians, American College of Sports Medicine, American Physical Therapy Association, Canadian Association of Cardiac Rehabilitation, European Association for Cardiovascular Prevention and Rehabilitation, Inter-American Heart Foundation, National Association of Clinical Nurse Specialists, Preventive Cardiovascular Nurses Association, and the Society of Thoracic Surgeons. J Am Coll Cardiol. 2007;50:1400-1433.

5. Welker J, Nacht CA, Savcic M, et al. Cardiac rehabilitation, a proved benefic intervention underused in the French-speaking part of Switzerland. Rev Med Suisse. 2014;10:545548.

6. Mihaela Mocan, Roxana Chiorescu, Oana Natalia Banc, Bogdan Mocan, Florin Anton, Mirela Stoia, Anca Daniela Farcas. Cardiac rehabilitation protocols outcome in frail patients undergoing transcatheter aortic valve implantation. Balneo Research Journal. 2019:9:401-405.

7. Oana Moldovan, Balasz Deak, Alin Bian, Diana Gurzau, Florina Frangu, Alexandru Martis, Bogdan Caloian, Horatiu Comsa, Gabriel Cismaru, Dana Pop How do I track Cardiac Rehabilitation in my patient with ischemic heart disease using Strava. Balneo Research Journal. 2019:10:114-117.

8. Chan Keith Andrew, Cismaru Gabriel, Istratoaie Sabina, Puiu Mihai, Gusetu Gabriel, Caloian Bogdan, Comsa Horatiu, Frangu Florina, Gurzau Diana, Martis Alexandru, Pop Dana, Zdrenghea Dumitru, Rosu Radu. Cardiac rehabilitation in elderly athletes. Analysis of elderly participation in Wizz Air Cluj-Napoca Marathon. Balneo Research Journal. 2019:10:231-235.

9. Mihaela Mocan, Bogdan Mocan. Cardiac rehabilitation for older patients with cardiovascular pathology using robotic systems - A survey. Balneo Research Journal 2019:10:33-36.

10. Bogdan Caloian, Gabriel Nicolae Guşetu, Simona Costea, Dan Horaţiu Comsa, Gabriel Laurenţiu Cismaru, Radu Ovidiu Roşu, Dana Pop, Dumitru Tudor Zdrenghea. The effect of cardiac pacemaker implantation on cardiac performance - the experience of a Cardiology - Rehabilitation Department. Balneo Research Journal. 2019:9:54-58. 
11. Bogdan Caloian, Dana Pop, Gabriel Guşetu, Dumitru Zdrenghea. The role of cardiopulmonary exercise testing in the initial evaluation of patients wearing intracardiac devices submitted to cardiac rehabilitation. 2017:8:206-211.

12. Salazar Adum JP, Rohit A. Treatment for Inappropriate Sinus Tachycardia. American Journal of Therapeutics .2015;10, 1-5.

13. Femenía F, Baranchuk A, MD, Morillo CA. Inappropriate Sinus Tachycardia: Current Therapeutic Option. Cardiology in Review. 2012;20: 8-14.

14. Lillis DL, Hanson P. Ventricular ectopy in cardiac rehabilitation patients on exercise training and nonexercising days. Clin. Cardiol. 1989;12:569-574.

15. Marouane B, Salvatore DT, Rym K. Impacts of cardiac rehabilitation on ventricular repolarization indexes and ventricular arrhythmias in patients affected by coronary artery disease and type 2 diabetes. Heart and Lung. 2015;44:199-204.

16. Jo Kato, Akira Koike, Kenji Kuroki, et al. Safety and efficacy of in-hospital cardiac rehabilitation following antiarrhythmic therapy for patients with electrical storm. Journal of Cardiology. 2019,73:171-178.

17. Relation of Atrial Premature Complexes During Exercise Stress Testing to the Risk for the Development of Atrial Fibrillation in Patients Undergoing Cardiac Rehabilitation. Am J Cardiol. 2018;122:395-399.

18. Sabina Istratoaie, Gabriel Cismaru, Radu Rosu, Alin Bian, Diana Gurzău, Florina Frîngu, Bogdan Caloian, Horaţiu Comșa, Alexandru Marțiș, Gabriel Gușetu, Dumitru Zdrenghea, Dana Pop, Anca Buzoianu. Cardiac rehabilitation after catheter ablation of atrial fibrillation. Balneo Research Journal .2019:9:33-36.335-339.

19. Mozaffarlan D, Furberg CD, Psaty BM, Siscovick D. Physical Activity and Incidence of Atrial Fibrillation in Older Adults: The Cardiovascular Health Study: MozaffarianPhysical activity and atrial fibrillation. Circulation. 2008;118:800-7.
20. Rajeev K Pathak, Adrian Elliott, Melissa E. Middeldorp, Megan Meredith, Abhinav B. Mehta M. Impact of CARDIOrespiratory FITness on Arrhythmia Recurrence in Obese Individuals With Atrial Fibrillation: The CARDIO-FIT StudyJournal of the American College of Cardiology. 2015;66:985-996.

21. Osbak PS, Mourier M, Kjaer A, Henriksen JH, Kofoed KF, Jensen GB. A randomized study of the effects of exercise training on patients with atrial fibrillation. Am Heart $\mathbf{J}$ .2011;162:1080-1087.

22. Plisiene J1, Blumberg A, Haager G, Knackstedt C, Latsch J, Norra C, Arndt M, Tuerk S, Heussen N, Kelm M, Predel HG, Schauerte P. Moderate physical exercise: a simplified approach for ventricular rate control in older patients with atrial fibrillation. Clin Res Cardiol. 2008 ;97:820-6.

23. Myrstad M, Nystad W, Graff-Iversen S et al. Effect of Years of Endurance Exercise on Risk of Atrial Fibrillation and Atrial Flutter. Am J Cardiol. 2014;114:1229-1233. 